Original Article

\title{
Effects of push-up exercise on shoulder stabilizer muscle activation according to the grip thickness of the push-up bar
}

\author{
JAemin Jung, $\mathrm{PhD}^{1)}$, Woonik Cho, $\mathrm{PhD}^{2)^{*}}$ \\ 1) Department of Physical Therapy, College of Science, Kyungsung University, Republic of Korea \\ 2) Division of English Interpretation and Translation Studies, Busan University of Foreign Studies: \\ Namsan-dong, Geumjeong-gu, Busan 609-815, Republic of Korea
}

\begin{abstract}
Purpose] This study investigated the effects of bar thickness on shoulder stabilizer muscle activation during push-up exercise. [Subjects] Twenty-six healthy male adults in their twenties. [Methods] The study had four experimental conditions (grip thicknesses of $0 \%, 50 \%, 75 \%$, and $100 \%$ of the subjects' hand size). Measurements were conducted from the start to the end of push-up for deltoid anterior fiber, deltoid posterior fiber, infraspinatus, serratus anterior, and pectoralis major muscle activation. [Results] The deltoid anterior fiber muscle activity was $4,852.6 \pm 975.2$ in the $0 \%, 5,787.3 \pm 1,514.1$ in the $50 \%, 5,635.3 \pm 1,220.1$ in the $75 \%$, and $5,032.9 \pm 841.0$ in the $100 \%$ condition. The infraspinatus muscle activity was $1,877.2 \pm 451.3$ in the $0 \%, 2,310.9 \pm 765.4$ in the $50 \%, 2,353.6 \pm$ 761.9 in the $75 \%$, and $2,016.8 \pm 347.7$ in the $100 \%$ condition. The pectoralis major muscle activity was $1,675.8 \pm$ 355.1 in the $0 \%, 2,365.5 \pm 1,287.3$ in the $50 \%, 2,125.3 \pm 382.5$ in the $75 \%$, and $1,878.8 \pm 419.7$ in the $100 \%$ condition, showing significant differences respectively. [Conclusion] The use of push-up bars with different thicknesses customized to personal characteristics, rather than the conventional standard, could be more effective for training and rehabilitation.
\end{abstract}

Key words: Electromyogram, Push-up bar, Shoulder stabilizer muscle

(This article was submitted Jun. 3, 2015, and was accepted Jun. 24, 2015)

\section{INTRODUCTION}

Pain and dysfunction of the shoulder joint is one of the most common musculoskeletal disorders ${ }^{1)}$. The balance among stabilizer muscles is considered important in therapeutic exercise programs for the prevention and rehabilitation of a dysfunctional shoulder joint. Moreover, several types of rehabilitation programs are available for patients. From the aspect of motor mechanics, a closed kinetic chain exercise is often used ${ }^{2}$.

The push-up exercise is a representative closed kinetic chain exercise of the upper extremity. It is an easy exercise for strengthening the muscles around the shoulder and is hence widely used as a general therapeutic exercise for improving the shoulder function of individuals with shoulder problems ${ }^{3)}$. Many studies have examined the enhancement of shoulder joint muscle activation during the push-up exercise. Compared with a push-up exercise on a stable surface, a push-up exercise on an unstable surface increases the activity of the shoulder stabilizer muscles and effectively

*Corresponding author. Woonik Cho (E-mail: cloud@bufs. ac.kr)

C2015 The Society of Physical Therapy Science. Published by IPEC Inc. This is an open-access article distributed under the terms of the Creative Commons Attribution Non-Commercial No Derivatives (by-ncnd) License $<$ http://creativecommons.org/licenses/by-nc-nd/3.0/>. improves balance through the stimulation of muscle proprioceptors during rehabilitation exercise ${ }^{4}$.

Lee et al. reported strong activation of the shoulder stabilizer muscles, even when a small weight load is used, during abduction-adduction via distal flexor muscle activation. They also observed that movement with finger flexor muscle activation had a substantial effect on the activation of the shoulder stabilizer muscles ${ }^{5}$. . Another study indicated that a push-up plus exercise using a push-up bar was more effective than that performed on flat ground. The push-up bar exercise increased the muscle activation of the forearm flexor bundle for gripping, which further led to increased activation of the shoulder stabilizer muscles ${ }^{6}$. These findings suggest that along with the use of various support surfaces during the push-up exercise for the rehabilitation of the shoulder joint, the use of a push-up bar is another effective method for both stimulating the proprioceptors and increasing the muscle activation of the shoulder stabilizer muscles.

However, previous studies have investigated the effects of the push-up exercise using the push-up bar on shoulder stabilizer muscle activation, solely based on grip direction and use. Hence, the effects of the grip thickness of the pushup bar on shoulder stabilizer muscle activation are unclear. This present study aimed to examine the effects of the grip thickness of the push-up bar on shoulder stabilizer muscle activation during push-up exercise. 


\section{SUBJECTS AND METHODS}

The subjects were 26 male adults in their twenties who had no low back pain or musculoskeletal disease of the shoulder complex and upper limbs, had a normal range of motion, and could perform push-ups. This research complied with the ethical principles of the Declaration of Helsinki. All of the participants who voluntarily participated in the research experiment fully understood the experimental procedure and method before participation. In particular, all of the subjects read, understood, and signed the written consent form distributed. In addition, none of the processes in this research was harmful to the human body. The age, weight, and height (mean $\pm \mathrm{SD}$ ), and hand length (mean) of the subjects were $22.16 \pm 2.23$ year, $68.02 \pm 10.86 \mathrm{~kg}$, and $171.22 \pm 3.58 \mathrm{~cm}$, and $19.2 \mathrm{~cm}$, respectively.

In each condition, the push-up was started at a crawling position, with both hands on the floor or holding a push-up bar grip at shoulder width and the third finger below the acromioclavicular joint. When holding the grip, each subject was guided to seize the grip as strongly as possible by using the forearm muscles. After the subject's shoulder was accurately positioned, both feet were placed close together, with the knees in a comfortable position and the pelvis in a neutral position ${ }^{7}$.

The experiments were conducted under four conditions. The first condition was $0 \%$, and the subjects performed the push-up exercise on the 10 -cm high box in order to make the same height as that of the push-up bar. The second condition was $50 \%$. The subjects performed the push-up exercise with a grip thickness of $50 \%$ of their hand size. The third condition was $75 \%$. The subjects performed the push-up exercise with a grip thickness of $75 \%$ of their hand size. The fourth condition was $100 \%$. The subjects performed the push-up exercise with a grip thickness of $100 \%$ of their hand size. Their hand size was determined as the length from the proximal point of the lunate to the end of the distal phalanx of the third finger and set as $100 \%$. The push-up bar was manufactured by Nike Inc. (EFO114-085, USA). The grip thickness was adjusted in line with each condition $(50 \%, 75 \%$, or $100 \%)$ by winding a taping tape around the push-up bar grip according to the hand size measured.

To measure muscle activation in the shoulder stabilizer muscles, electrodes were attached to the deltoid anterior fiber, deltoid posterior fiber, infraspinatus, serratus anterior, and pectoralis major. Muscle activation was measured by the researcher from the start to the end of one push-up. Muscle activation was measured from the start to the end of a push-up exercise. The exercise was repeated three times under each condition, and the average of the three measurements was determined. The effects of muscle fatigue were prevented by randomly arranging the conditions $(0 \%, 50 \%, 75 \%$, or $100 \%$ ) and allowing a 5-min rest between each condition. Electromyography (EMG) was performed after depilating with a razor the parts for electrode attachment, removing the horny layer with sand paper, and cleansing the area with an alcohol swab to collect accurate data. ProComp Infiniti (Thought Technology Ltd., Canada) was used for measurement of muscle activation. A surface electrode (Triode, Thought Technology Ltd.) consisting of a tripolar electrode (positive-ground-negative) was used. The frequency range of the electromyographic signals was set to 20 to $500 \mathrm{~Hz}$, and the sampling frequency was set to $1,024 \mathrm{~Hz}$.

The root mean square values of each muscle were measured for 5 seconds in the anatomical position. The relative muscle contraction was calculated with respect to the mean EMG signal for 3 seconds in the middle portion of the muscle, excluding the measurements for the first and last seconds. The muscle activation resulting from one push-up was expressed as the relative muscle contraction in \% RVC.

PASW (Ver. 19) for Windows was used for data analysis. In order to compare shoulder stabilizer muscle activation between the push-up grip thicknesses, one-way analysis of variance was performed. Meanwhile, the Tukey test was used as a post hoc test to verify the differences between each condition. The level of significance was set at $\mathrm{p}=0.05$.

\section{RESULTS}

The deltoid anterior fiber muscle activity was $4,852.6 \pm$ 975.2 in the $0 \%, 5,787.3 \pm 1,514.1$ in the $50 \%, 5,635.3 \pm$ $1,220.1$ in the $75 \%$, and $5,032.9 \pm 841.0$ in the $100 \%$ condition, showing differences respectively. The post hoc test revealed significant differences between the $0 \%, 75 \%, 100 \%$, and $50 \%$ conditions $(\mathrm{p}<0.05)$. Infraspinatus muscle activity was $1,877.2 \pm 451.3$ in the $0 \%, 2,310.9 \pm 765.4$ in the $50 \%$, $2,353.6 \pm 761.9$ in the $75 \%$, and $2,016.8 \pm 347.7$ in the $100 \%$ condition. The post hoc test showed significant differences between the $0 \%, 50 \%, 100 \%$, and $75 \%$ conditions $(\mathrm{p}<0.05)$. The pectoralis major muscle activity was $1,675.8 \pm 355.1$ in the $0 \%, 2,365.5 \pm 1,287.3$ in the $50 \%, 2,125.3 \pm 382.5$ in the $75 \%$, and $1,878.8 \pm 419.7$ in the $100 \%$ condition, showing significant differences as well. The post hoc test showed significant differences between the $0 \%, 75 \%, 100 \%$, and $50 \%$ conditions $(\mathrm{p}<0.01$; Table 1$)$.

\section{DISCUSSION}

Lee et al. reported that the stimulation of the distal part due to the weight load on the forearm during shoulder joint movement caused strong activation of the shoulder stabilizer muscles. This finding indicated that the activation of the finger flexor had a major effect on shoulder muscle activation $^{5)}$. They also reported that for shoulder stabilizer muscle activation, a push-up exercise with a push-up bar was more effective than a push-up exercise on a flat floor owing to the involvement of the forearm finger flexor ${ }^{6)}$. This present study aimed to identify the effects of push-up bar thickness on shoulder stabilizer muscle activation during push-up exercise.

Lee et al. observed forearm muscle activation at grip thicknesses of $0 \%, 25 \%, 50 \%, 75 \%$, and $100 \%$. They reported that maximum muscular activity occurred at grip thickness of $50 \%$ and $75 \%$ for the wrist extensor bundle, and at grip thickness of $75 \%$ for the flexor digitorum superficialis. These results indicated the close relationship of the length-tension curve ${ }^{7)}$.

The conditions in the present study were similar to those used by Lee et al.7), namely grip thicknesses of $0 \%, 50 \%$, $75 \%$, and $100 \%$. However, a thickness of $25 \%$ was not used 
Table 1. Muscle activation (\%RVC) under the push-up conditions

\begin{tabular}{lllll}
\hline Muscle & \multicolumn{1}{c}{$0 \%$} & \multicolumn{1}{c}{$50 \%$} & \multicolumn{1}{c}{$75 \%$} & $100 \%$ \\
\hline Deltoid anterior fiber* & $4,852.6 \pm 975.2^{\mathrm{a}}$ & $5,787.3 \pm 1,514.1^{\mathrm{b}}$ & $5,635.3 \pm 1,220.1^{\mathrm{a}}$ & $5,032.9 \pm 841.0^{\mathrm{a}}$ \\
Deltoid posterior fiber & $2,023.2 \pm 1,097.2$ & $2,642.6 \pm 1,121.6$ & $2,597.0 \pm 1,112.2$ & $2,172.0 \pm 1,155.9$ \\
Infraspinatus* $^{\text {Pectoralis major* }}$ & $1,877.2 \pm 451.3^{\mathrm{a}}$ & $2,310.9 \pm 765.4^{\mathrm{a}}$ & $2,353.6 \pm 761.9^{\mathrm{b}}$ & $2,016.8 \pm 347.7^{\mathrm{a}}$ \\
Serratus anterior & $1,675.8 \pm 355.1^{\mathrm{a}}$ & $2,365.5 \pm 1,287.3^{\mathrm{b}}$ & $2,125.3 \pm 382.5^{\mathrm{a}}$ & $1,878.8 \pm 419.7^{\mathrm{a}}$ \\
\hline
\end{tabular}

The data represent mean \pm SD values. The values with different superscripts in the same column were significantly different $(\mathrm{p}<0.05)$ in the Tukey analysis. ${ }^{\mathrm{a}}$ : Significant difference between $0 \%$ and $50 \%,{ }^{\mathrm{b}}$ : Significant difference between $0 \%$ and $75 \%$

in the present study, as the thickness of the push-up bar was approximately $45 \%$ of the subjects' hand size. In the present study, a significantly high muscular activity was noted at the deltoid anterior fiber and pectoralis major at a push-up bar grip thickness of $50 \%$, and at the infraspinatus at a push-up bar grip thickness of $75 \%$. In addition, high muscular activity was observed at the deltoid posterior fiber and serratus anterior at a push-up bar grip thickness of $50 \%$ and $75 \%$, although this increase was not significant. These results indicate that a push-up bar grip thickness of $50 \%$ and $75 \%$ was effective for the activation of the forearm muscles during push-up exercise, which may have consequently influenced the activation of the shoulder stabilizer muscles during the push-up exercise.

The present study findings indicate that the effects of the exercise could be improved simply by changing the grip thickness of the distal part in order to induce strong activation of the muscles of the proximal part. Thus, the use of push-up bars with a thickness customized according to individuals' characteristics could be more effective for training and rehabilitation than the use of a conventional standard push-up bar.

Further studies are required to verify the effect of grip thickness of standard elastic bands, dumbbells, or barbells in open kinetic chain exercises.

\section{ACKNOWLEDGEMENT}

This research was supported by the research grant of the Busan University of Foreign Studies in 2015.

\section{REFERENCES}

1) Andrews JR: Diagnosis and treatment of chronic painful shoulder: review of nonsurgical interventions. Arthroscopy, 2005, 21: 333-347. [Medline] [CrossRef]

2) Ludewig PM, Hoff MS, Osowski EE, et al.: Relative balance of serratus anterior and upper trapezius muscle activity during push-up exercises. Am J Sports Med, 2004, 32: 484-493. [Medline] [CrossRef]

3) Lunden JB, Braman JP, Laprade RF, et al.: Shoulder kinematics during the wall push-up plus exercise. J Shoulder Elbow Surg, 2010, 19: 216-223. [Medline] [CrossRef]

4) Kim M, Jung J, Lee S, et al.: Effect of various lower limb ground states on activation of the shoulder and trunk muscles during push-up exercise. J Phys Ther Sci, 2012, 24: 161-164. [CrossRef]

5) Lee SY, Gong WT, Park MC, et al.: A study of shoulder stabilizer muscle exercise using contraction of the finger flexor muscle. J Phys Ther Sci, 2011, 23: 41-43. [CrossRef]

6) Lee SY, Jung JM, Hwangbo G: The effects on shoulder stabilizer activation of finger flexor activation during the push-up plus exercise. J Phys Ther Sci, 2011, 23: 575-577. [CrossRef]

7) Lee S, Shim J, Park M: A study on the activation of forearm muscles during gripping by handle thickness. J Phys Ther Sci, 2011, 23: 549-551. [CrossRef] 